\title{
The Cataract Explosion: The Changing Pattern of Diagnoses of Patients Attending an Ophthalmic Outpatient Department
}

\author{
M. BATTERBURY, P. T. KHAW, R. HANDS, A. R. ELKINGTON \\ Southampton
}

\begin{abstract}
Summary
An outpatient diagnostic index was used to analyse data relating to a sample of new patients attending the Southampton Eye Hospital in 1979 and 1986. Our main findings are an increased demand for cataract surgery by an increasingly aged population and a trend towards operating on cataracts at an earlier stage in their development. These and other results are discussed.
\end{abstract}

The collection and analysis of data relating to the provision of health care have always been of great importance in the planning of services but have recently received wider attention with the debate of the Government's proposed reforms of the National Health Service. Additionally, changes in the pattern of demands for health care need to be recognised if appropriate changes in the provision of services are to be made. Whilst data relating to hospital inpatients have been collected for some time, less assessment of the work of outpatient departments has been performed. Specialties such as ophthalmology have a particularly heavy outpatient load, and are associated with long delays for new appointments.

The Southampton Eye Hospital established an outpatient diagnostic index in $1977^{1}$ and has surveyed the services of its Accident and Emergency Department. ${ }^{2}$ Others have examined the work of ophthalmic outpatient and casualty units, ${ }^{3.4 .5}$ but to date there has been no published analysis of the changing patterns in demand on the services of an ophthalmic outpatient department.
The aim of this study was to review the details of patients referred to the Southampton Eye Hospital Outpatient Department in two contrasting years, 1979 and 1986.

\section{Methods}

Patient names and index numbers were obtained from clinic lists for 1979 and 1986, new patient lists being kept separate from those for old patients. New patients are those referred for the first time or referred again with a new or recurrent problem. The case notes of a sample of one-in-five of these were obtained and examined, and relevent data entered onto a computer database (Reflex, Borland) on an IBM PC compatible computer. In cases where the diagnosis was uncertain or had not been recorded, the notes were re-examined by one of us (MB) before definitive diagnostic coding. The diagnostic index used was Series 9 of the International Classification of Disease. The setting-up of this index has previously been described. ${ }^{1}$

\section{Results}

A total of 828 and 1055 case notes for the 
years 1979 and 1986 respectively were examined, but 71 from 1979 were not traced (Table I). This would suggest an annual new patient attendance of 4,495 for 1979 and 5,295 for 1986 . This is an increase in 1986 of $18 \%$ on 1979. These are underestimates, data from other hospital sources indicating that 4,979 new patients attended in 1979 and 5,693 in 1986 (an increase of 14\%).

Much of this increase was due to an increase in the number of patients aged over 60 years.

Table II summarises the main diagnostic data. A total of 186 different diagnoses were made on this sample, and many patients had more than one diagnosis at presentation. Only those diagnoses made at least ten times are tabulated. Certain features of these data will be discussed later.

Table III shows the referring source and Table IV details of the wait between referral and attendance in the Department. Table V summarises the data relating to listing for surgery. Whilst there has been no increase in the proportion of new patients listed for surgery at their first visit, there is an increase in the proportion of those listed who are to have cataract extraction (23\% 1979, 39\% 1986) and in those whose main diagnosis is senile cataract who are judged suitable for surgery $(22 \%$ $1979,47 \%$ 1986). There was also a trend in 1986 to operate on patients with cataract at an earlier stage (i.e. with better visual acuity at presentation) (Fig. 1).

The largest proportion of patients listed for surgery at their first visit were to have minor procedures on the eyelids (48\% 1979, 43\% 1986). These procedures were usually performed under local anaesthesia.

\section{Discussion}

The introduction of a diagnostic index is

Table I

\begin{tabular}{lrrrr}
\hline & \multicolumn{2}{c}{1979} & \multicolumn{1}{c}{1986} \\
\hline Case notes traced & 828 & 1,055 & \\
Case notes not traced & 71 & 4 & \\
Estimated total new & & & & \\
$\quad$ patients & 4,495 & 5,295 & \\
Actual new patients & 4,979 & 5,693 & \\
Age < 10 years & 205 & $(25 \%)^{2}$ & 271 & $(26 \%)$ \\
Age $>60$ years & 263 & $(32 \%)$ & 423 & $(40 \%)$ \\
\hline
\end{tabular}

' Figures obtained from other hosptial records.

- Percentages are those of notes traced. essential in the analysis of outpatient work, and will help with the practice of medical audit. Data presented here are the first to be published comparing the work of an ophthalmic outpatient department in two different years.

A study such as this is subject to several sources of error: in the encoding of diagnoses, in the transcription of data onto a computer database, and in the retrieval of case notes. The estimates for new attenders is less than the number known from other hospital records to have attended (Table I), and is probably accounted for by our use of clinic appointment lists as our source of patient names and numbers. Some new patients may be seen in 'old patient' clinics and not be entered onto new patient clinic lists, such as some patients referred from the Eye Casualty Department and patients seen by junior staff at other hospitals and followed-up in the outpatient department.

There are certain trends from 1979 to 1986 that require consideration. The increase in the load of new patients and the greater proportion aged over 60 years (Table I) mirror the increase in size of the elderly population and recent improvements in ophthalmic care, particularly relating to cataract and laser surgery. Similarly the increased presentation of pre-senile cataract, glaucoma, glaucoma suspects and age-related macular degeneration may reflect public, general practitioner and optometrist awareness of the advancement of ophthalmic treatments. It is unlikely that it represents increased incidence (although data

Table II Diagnostic details (\% of notes traced)*

\begin{tabular}{lrr}
\hline & 1979 & 1986 \\
\hline Nothing abnormal detected & 8.3 & 8.5 \\
Ocular-motility disturbance & 19.3 & 12.2 \\
Cataract & 12.1 & 12.3 \\
Refractive error & 7.1 & 10.6 \\
Glaucoma & 4.9 & 9.4 \\
Chalazion & 3.6 & 4.1 \\
Tear drainage impairment & 3.3 & 2.6 \\
Vitreous abnormalities & 3.1 & 2.7 \\
Dry eye & 3.0 & 2.4 \\
Blepharitis & 2.5 & 2.0 \\
Age-related macular degeneration & 2.2 & 2.9 \\
Retinal vascular occiusion & 1.8 & 1.2 \\
Diabetic retinopathy & 1.2 & 0.9 \\
\hline
\end{tabular}

* Only diagnoses made at least ten times are shown. 
Table III Source of referral

\begin{tabular}{lrr}
\hline & 1979 & 1986 \\
\hline General practitioner & $567(69 \%)$ & $791(75 \%)$ \\
SEH $^{1}$ A and E Dept & $63(8 \%)$ & $58(6 \%)$ \\
General practitioner/ & & \\
$\quad$ SEH A and E & $45(5 \%)$ & $43(4 \%)$ \\
Other & $153(18 \%)$ & $163(15 \%)$ \\
\hline
\end{tabular}

${ }^{1}$ Southampton Eye Hospital

${ }^{2}$ Patients referred from the GP via the A and E Dept.

relating to true incidence of ocular conditions in the general population are scant) since causes of blind and partial sight registration have remained largely unchanged. ${ }^{6}$

The proportion of new patients listed for cataract surgery has increased (Table V) and the decision to perform surgery is taken at an earlier stage in visual loss, again reflecting improved success in cataract surgery. This has implications for post-operative care, as at least three post-operative visits will be required. Indeed, some ophthalmologists would recommend that patients with an intraocular lens implant should never be discharged from hospital care. On the other hand, patients being referred earlier in the development of their cataract but not suitable for surgery at presentation may require subsequent assessment in the clinic.

Jay and Devlin ${ }^{7}$ also found an increase in cataract surgery, particularly amongst the elderly, and also noted a tendency to operate on eyes at a better level of visual acuity. Such changes need to be recognised in the planning of future ophthalmic services.

The number of patients with suspected glaucoma increased between 1979 and 1986. Harrison et al. ${ }^{8}$ found suspected glaucoma to be the second most common reason for referral. Improved detection of patients with glaucoma, or increased referral of glaucoma suspects requiring regular review, will also throw a greater load on old patient clinics.

Table IV Waiting time for outpatient appointment (weeks)

\begin{tabular}{lcc}
\hline & 1979 & 1986 \\
\hline Average wait & 9.5 & 9.7 \\
Range & $0-46$ & $0-56$ \\
Range of average wait by consultant & $6-13$ & $7-11$ \\
\hline
\end{tabular}

The extension of the diagnostic index to old patients would help to clarify such trends, but it has not been possible to achieve this.

A large portion of new patient work involves minor lid pathology and surgery (Tables II and V). Diminution in this work would considerably lighten new patient clinics.

General practitioners are still the main source of referral (Table III), though our data did not differentiate between patients referred from optometrists via the GP and those referred without prior optometric assessment. Our figures do not show any greater tendency for the casualty department to be used as a short-cut into the outpatient department.

We have recorded a large number of patients with no abnormality detected (Table II). The debate concerning the abilities of optometrists and general practitioners to recognise ocular pathology continues. ${ }^{8}$

The diagnostic index at the Leicester Royal Infirmary was used by Shaw et al. ${ }^{3}$ to examine the records of new and old patients, but outpatient casualty patients and children were excluded. Their population of new patients was thus different from our own. Further analysis of our data shows that lens-related conditions account for $12 \%$ of diagnoses, and that $16 \%$ of patients in 1979 and $13 \%$ in 1986 had one of the four major blinding conditions (senile cataract, age-related macular degeneration, primary open angle glaucoma and diabetic retinopathy). These are very different from Shaw's figures of $31 \%$ and $45 \%$ respectively. In addition, they note a higher proportion listed for surgery at first visit, 17\%

Table V Patients listed for surgery at first visit

\begin{tabular}{lrr}
\hline & 1979 & 1986 \\
\hline $\begin{array}{l}\text { Number listed for surgery (\% } \\
\text { of new attenders) }\end{array}$ & & \\
$\begin{array}{l}\text { Procedure } \\
\text { cataract }\end{array}$ & $23(12 \%)$ & $137(13 \%)$ \\
strabismus & $12(12 \%)$ & $54(39 \%)$ \\
I and C lid cyst & $26(26 \%)$ & $41(30 \%)$ \\
excision of lid lump & $13(13 \%)$ & $9(6 \%)$ \\
other minor lid procedures & $9(9 \%)$ & $10(7 \%)$ \\
other & $18(18 \%)$ & $15(11 \%)$ \\
$\%$ of patients presenting with & & \\
senile cataract listed at first & & \\
visit & 22 & 47 \\
\hline
\end{tabular}




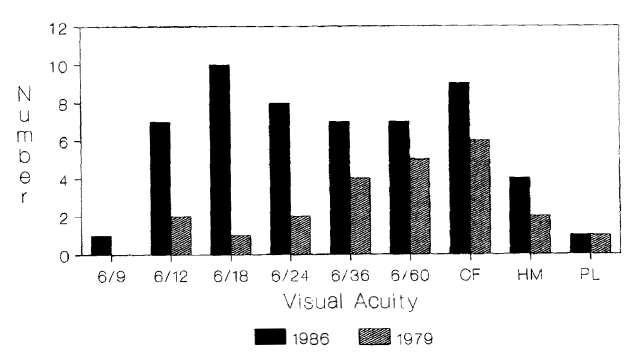

Fig. 1. Visual acuity at listing for cataract extraction.

compared with our $12-13 \%$. These and other discrepancies may. relate to their different population and our recording of more than one diagnosis in some patients (which may over-emphasise secondary and tertiary diagnoses). In addition ours was a sample, whereas Shaw etal. examined data on all patients.

It is important to note that diagnostic frequencies presented here do not relate to incidence of ocular conditions in the general population. However Brennan and Knox ${ }^{9}$ used outpatient data to develop a concept of 'demand incidence', that is, the incidence of ocular disease requiring assessment and treatment. Our figures suggest an increase in the demand on the Southampton Eye Hospital by patients aged over 60 years and by patients with cataract.

We thank the Wessex Regional Health Authority for financing the staff and computer software and hard- ware necessary for this study, and the consultant ophthalmologists and medical records staff at Southampton Eye Hospital for their cooperation.

Key words: diagnosis, medical audit, outpatient clinics, hospital records.

\section{References}

${ }^{1}$ Elkington AR and Davies JC: Setting up an outpatient diagnostic index. J Roy Soc Med 1980, 73: 646-8.

${ }^{2}$ Jones NP, Hayward JM, Khaw PT, Claoué CMP, Elkington AR: Functions of an Ophthalmic 'Accident and Emergency' Department: Results of a 6 month survey. Br Med J 1986, 292: 188-90.

${ }^{3}$ Shaw DE, Gibson JM, Rosenthal AR: A year in a general ophthalmic outpatient department in England. Arch Ophthalmol 1986, 104: 1843-6.

${ }^{4}$ Chiapella AP and Rosenthal AR: One year in an eye casualty clinic. Br J Ophthalmol 1985, 69: 865-70.

${ }^{5}$ Vernon SA: Analysis of all new cases seen in a busy regional centre ophthalmic casualty department during 24-week period. J Roy Soc Med 1983, 76: 279-82.

${ }^{6}$ Grey RHB, Burns-Cox CJ, Hughes A: Blind and partial sight registration in Avon. $\mathrm{Br} J$ Ophthalmol 1989, 73: 88-94.

${ }^{7}$ Jay JL and Devlin ML: The increasing frequency of surgery for cataract. Eye 1990, 4: 127-31.

${ }^{8}$ Harrison RJ, Wild JM, Hobley AJ: Referral patterns to an ophthalmic outpatient clinic by general practitioners and ophthalmic opticians and the role of these professionals in screening for ocular disease. Br Med J 1988, 297: 1162-7.

${ }^{9}$ Brennan ME and Knox EG: The incidence of cataract and its clinical presentation. Community Health 1975, 7: 13-20. 